\title{
ATTRACTORS AND ORBIT-FLIP HOMOCLINIC ORBITS FOR STAR FLOWS
}

\author{
C. A. MORALES
}

(Communicated by Bryna Kra)

\begin{abstract}
We study star flows on closed 3-manifolds and prove that they either have a finite number of attractors or can be $C^{1}$ approximated by vector fields with orbit-flip homoclinic orbits.
\end{abstract}

\section{INTRODUCTION}

The notion of attractor deserves a fundamental place in the modern theory of dynamical systems. This assertion, supported by the nowadays classical theory of turbulence [27], is enlightened by the recent Palis conjecture [24] about the abundance of dynamical systems with finitely many attractors absorbing most positive trajectories. If confirmed, such a conjecture means the understanding of a great part of dynamical systems in the sense of their long-term behaviour.

Here we attack a problem which goes straight to the Palis conjecture: The finitude of the number of attractors for a given dynamical system. Such a problem has been solved positively under certain circunstances. For instance, we have the work by Lopes [16, who, based upon early works by Mañé [18] and extending previous ones by Liao [15] and Pliss [25], studied the structure of the $C^{1}$ structural stable diffeomorphisms and proved the finitude of attractors for such diffeomorphisms. His work was largely extended by Mañé himself in the celebrated solution of the $C^{1}$ stability conjecture [17. On the other hand, the Japanesse researchers S. Hayashi [11] and N. Aoki [2] studied star diffeomorphisms, i.e., diffeomorphisms which cannot be $C^{1}$ approximated by ones with nonhyperbolic periodic points, and proved that they are Axiom A and so with only a finite number of attractors. Their investigation triggered the study of the star flows, i.e., vector fields which cannot be $C^{1}$ approximated by ones with nonhyperbolic closed orbits. Indeed, although it was known from the very beginning that these flows are not necessarily Axiom A [1], 7], 8], the aforementioned works by Liao and Pliss proved that they display finitely many attracting closed orbits.

Progress toward understanding star flows was tackled in 2003 by the author in collaboration with Pacifico [20]. Indeed, these authors proved on closed 3-manifolds that, except in a meager set, all such flows are singular-Axiom A and so with only a finite number of attractors. Soon after the Chinese authors Gan and Wen [5] extended Aoki-Hayashi's conclusion to nonsingular star flows on closed manifolds,

Received by the editors July 28, 2001 and, in revised form, November 3, 2011.

2010 Mathematics Subject Classification. Primary 37D20; Secondary 37C10.

Key words and phrases. Star flow, attractor, orbit-flip homoclinic orbit.

The author was partially supported by CNPq, FAPERJ and PRONEX-Brazil. 
implying that these flows have a finite number of attractors too. In light of these works, it seems quite promising to prove the finiteness of the number of attractors for star flows in any closed manifold.

In this paper we shall provide a result which, though partial, provides an insight for a positive solution of this problem. Basically, we present the so-called orbit-flip homoclinic orbits as an obstruction for the finiteness of attractors of star flows on closed 3-manifolds. More precisely, we show that a star flow on a closed 3-manifold either has a finite number of attractors or can be $C^{1}$ approximated by vector fields exhibiting orbit-flip homoclinic orbits. Orbit-flip homoclinic orbits are very rich dynamical structures which have been studied during the past few decades [3], 13], [14, 22], 23, 28, 29]. Let us state this result in a precise way.

Hereafter $M$ will denote a compact connected boundaryless Riemannian manifold of dimension $n \geq 2$ (a closed $n$-manifold for short). We shall consider a $C^{1}$ vector field $X$ in $M$ together with its induced one-parameter group $X_{t}, t \in \mathbb{R}$, the so-called flow of $X$. The space of $C^{1}$ vector fields in $M$ comes equipped with the $C^{1}$-topology which, roughly speaking, measures the distance between vector fields and their corresponding derivatives.

The long-time behavior of a point $x \in M$ is often analyzed through its omegalimit set

$$
\omega(x)=\left\{y \in M: y=\lim _{n \rightarrow \infty} X_{t_{n}}(y) \text { for some sequence } t_{n} \rightarrow \infty\right\} .
$$

A compact invariant set is transitive if it coincides with the omega-limit set of one of its points, whereas, in this work, an attractor will be a transitive set of the form

$$
A=\bigcap_{t>0} X_{t}(U)
$$

for some neighborhood $U$ of it. The most representative example of attractors are the sinks, that is, hyperbolic closed orbits of maximal Morse index. Sometimes we use the term source, referring to a sink for the time reversal vector field $-X$.

A homoclinic orbit is a regular (i.e. nonsingular) trajectory $\Gamma=\left\{X_{t}(q): t \in \mathbb{R}\right\}$ which is biasymptotic to a singularity $\sigma$, namely,

$$
\lim _{t \rightarrow \pm \infty} X_{t}(q)=\sigma .
$$

We call it an orbit-flip as soon as the eigenvalues $\lambda_{1}, \lambda_{2}, \lambda_{3}$ of $\sigma$ are real and satisfy the eigenvalue inequalities $\lambda_{2}<0<\lambda_{3}<\lambda_{1}$ and $\Gamma \subset W^{u u}(\sigma)$, where $W^{u u}(\sigma)$, the strong unstable manifold [12, is the unique invariant manifold of $X$ which is tangent at $\sigma$ to the eigenspace associated to the eigenvalue $\lambda_{1}$ (cf. Figure 1).

With such definitions and notation we can state our result.

Main Theorem. A star flow on a closed 3-manifold either has a finite number of attractors or can be $C^{1}$ approximated by vector fields exhibiting orbit-flip homoclinic orbits.

The proof relies on recent results in the theory of star flows [5], together with some techniques resembling those in [19.

The Main Theorem motivates the obvious question as to whether star flows which can be $C^{1}$ approximated by vector fields with orbit-flip homoclinic orbits exist on any closed 3-manifold. Actually this is true, but, as the reader can see for himself [15], 19], 22], 25], the set of such flows constitutes a meager subset of star flows. We therefore conclude that every closed 3-manifold comes equipped 


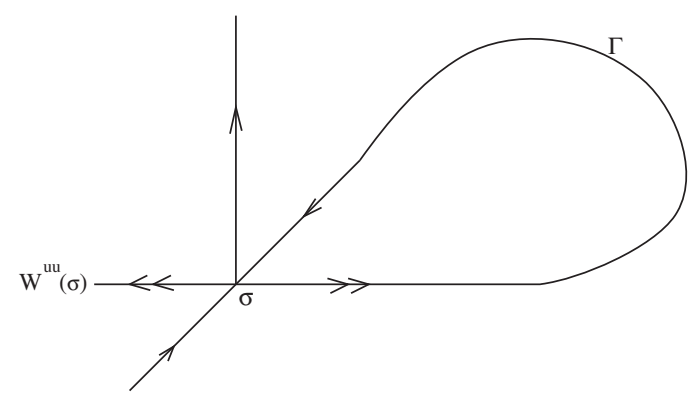

An orbit-flip homoclinic orbit

FiguRE 1

with an open and dense subset of star flows, all of whose elements have a finite number of attractors. However, it is worth noting that we can obtain exactly the same conclusion by making use of [19] and 20].

Another question is if, in the statement of the Main Theorem, we can replace the finitely many attractor's option by the stronger property of being singular-Axiom A (in the sense of [20]). Unfortunately, such a question has a negative answer, as we can easily find star flows in the 3 -sphere which are neither singular-Axiom A nor can be $C^{1}$ approximated by vector fields with orbit-flip homoclinic loops. Finally, let us mention that, in the statement of the Main Theorem, we can replace the term attractor by that of a Lyapunov stable omega-limit set (in the spirit of [21]).

\section{ProOF}

We denote by $\|\cdot\|$ the norm induced by a Riemannian metric in $M$ and by $m(\cdot)$ its corresponding minimum norm. Given a $C^{1}$ vector field $X$ with flow $X_{t}$ in $M$, we denote by $\operatorname{Sing}(X, U)$ the set of singularities of $X$ in $U$, and we write $\operatorname{Sing}(X)=\operatorname{Sing}(X, M)$. Likewise, the union of the periodic orbits of $X$ is denoted by $\operatorname{Per}(X)$. The elements of $\operatorname{Per}(X)$ will be called periodic points. A subset $\Lambda \subset M$ is called invariant if $X_{t}(\Lambda)=\Lambda$ for all $t \in \mathbb{R}$. A compact invariant set $\Lambda$ is hyperbolic if there are a tangent bundle decomposition $T_{\Lambda} M=\hat{E}_{\Lambda}^{s} \oplus E_{\Lambda}^{X} \oplus \hat{E}_{\Lambda}^{u}$ and positive constants $K, \lambda$ such that

- $\hat{E}_{\Lambda}^{s}$ is contracting, i.e.,

$$
\left\|D X_{t}(x) / \hat{E}_{x}^{s}\right\| \leq K e^{-\lambda t}, \quad \forall x \in \Lambda, t \geq 0 .
$$

- $\hat{E}_{\Lambda}^{u}$ is expanding, i.e.,

$$
m\left(D X_{t}(x) / \hat{E}_{x}^{u}\right) \geq K^{-1} e^{\lambda t}, \quad \forall x \in \Lambda, t \geq 0 .
$$

- $E_{\Lambda}^{X}$ is the subbundle generated by $X$ in $T_{\Lambda} M$.

Recall that a closed orbit $O$ (i.e. a periodic orbit or singularity) is hyperbolic if it is as a compact invariant set. A periodic point is hyperbolic if its corresponding orbit is hyperbolic. We say that a compact invariant set is nontrivial if it does not reduce to a single closed orbit.

For any given index $i$ in between 0 and $n-1$, we denote by $\operatorname{Per}_{i}(X)$ the union of the hyperbolic periodic orbits $O$ of $X$ with $\operatorname{dim}\left(\hat{E}_{x}^{s}\right)=i$ for some (and hence for all) $x \in O$. As pointed out earlier by Wen [30, we can extend this set to include 
periodic orbits for nearby vector fields. More precisely, we denote by $\operatorname{Per}_{i}^{*}(X)$ the $i$-preperiodic set of $X$ consisting of those $x \in M$ for which there are sequences $X_{k}$ and $x_{k}$ of vector fields and points in $\operatorname{Per}_{i}\left(X_{k}\right)$ such that $X_{k} \rightarrow X$ and $x_{k} \rightarrow x$. We shall also use the notion of a fundamental $i$-limit which are limits (in the Hausdorff metric) of sequences of hyperbolic periodic orbits $O_{n} \subset \operatorname{Per}_{i}\left(X_{n}\right)$ of vector fields $X_{n} \rightarrow X$.

Now we state four technical lemmas, the first of which is Lemma 3.4 in [5]:

Lemma 2.1. If $X$ is a star flow on a closed $n$-manifold and $\Lambda$ is a fundamental $i$-limit of $X$ with $\operatorname{Sing}(X, \Lambda)=\emptyset$, then $\Lambda$ is a sink or a source depending on whether $i=n-1$ or $i=0$.

Denote by $N \rightarrow M \backslash \operatorname{Sing}(X)$ the vector bundle with fiber $N_{x}=\left\{v \in T_{x} M\right.$ : $v \perp X(x)\}$. We define the linear Poincaré flow $P_{t}: N \rightarrow N$ by

$$
P_{t}=\pi \circ D X_{t},
$$

where $\pi: T M \rightarrow N$ stands for orthogonal projection. A $P_{t}$-invariant splitting over an invariant set $\Lambda \subset M \backslash \operatorname{Sing}(X)$ is a direct sum $N_{\Lambda}=\Delta_{\Lambda}^{1} \oplus \Delta_{\Lambda}^{2}$ such that $P_{t}\left(\Delta_{x}^{1}\right)=\Delta_{X_{t}(x)}^{1}$ and $P_{t}\left(\Delta_{x}^{2}\right)=\Delta_{X_{t}(x)}^{2}$ for all $x \in \Lambda$ and $t \in \mathbb{R}$.

We shall use the following Doering criterium for hyperbolicity 4]: A compact invariant set $\Lambda$ with $\operatorname{Sing}(X, \Lambda)=\emptyset$ is hyperbolic if and only if there is a $P_{t^{-}}$ invariant splitting $N_{\Lambda}=\Delta_{\Lambda}^{1} \oplus \Delta_{\Lambda}^{2}$ over $\Lambda$ such that $\Delta_{\Lambda}^{1}$ is contracting and $\Delta_{\Lambda}^{2}$ is expanding; i.e., there are positive constants $K, \lambda$ satisfying

$$
\left\|P_{t}(x) / \Delta_{x}^{1}\right\| \leq K e^{-\lambda t} \quad \text { and } \quad m\left(P_{t}(x) / \Delta_{x}^{2}\right) \geq K^{-1} e^{\lambda t} \quad \forall x \in \Lambda, \forall t \geq 0 .
$$

A dominated splitting for $P_{t}$ over $\Lambda$ is a $P_{t}$-invariant splitting $N_{\Lambda}=\Delta_{\Lambda}^{-} \oplus \Delta_{\Lambda}^{+}$for which there are positive constants $K, \lambda$ satisfying

$$
\frac{\left\|P_{t}(x) / \Delta_{x}^{-}\right\|}{m\left(P_{t}(x) / \Delta_{x}^{+}\right)} \leq K e^{-\lambda t}, \quad \forall(x, t) \in \Lambda \times \mathbb{R}^{+} .
$$

A dominated $\rho$-splitting for $P_{t}$ over $\Lambda$ is a dominated splitting $N_{\Lambda}=\Delta_{\Lambda}^{-} \oplus \Delta_{\Lambda}^{+}$such that $\operatorname{dim}\left(\Delta_{x}^{-}\right)=\rho, \forall x \in \Lambda$.

The following is Lemma 3.10 in [5].

Lemma 2.2. Let $X$ be a star flow on a closed $n$-manifold and $\Lambda$ be a compact invariant set with $\operatorname{Sing}(X, \Lambda)=\emptyset$ for which there is a dominated $\rho$-splitting $N_{\Lambda}=$ $\Delta_{\Lambda}^{-} \oplus \Delta_{\Lambda}^{+}$for $P_{t}$ over $\Lambda$ with $1 \leq \rho \leq n-2$. If $\Delta_{\Lambda}^{-}$is not contracting, there is a fundamental $r$-limit contained in $\Lambda$ with $r<\rho$. Likewise, if $\Delta_{\Lambda}^{+}$is not expanding, there is a fundamental $r$-limit contained in $\Lambda$ with $r>\rho$.

The proof of the following result can be obtained as in Theorem 3.8 of [31] (see also the proof of Lemma 2.8 in [6]).

Lemma 2.3. If $X$ is a star flow, then for every index $1 \leq i \leq n-2$ there is a dominated $i$-splitting

$$
N_{\operatorname{Per}_{i}^{*}(X) \backslash \operatorname{Sing}(X)}=\Delta_{\operatorname{Per}_{i}^{*}(X) \backslash \operatorname{Sing}(X)}^{-} \oplus \Delta_{P e r_{i}^{*}(X) \backslash \operatorname{Sing}(X)}^{+}
$$

for $P_{t}$ over $\operatorname{Per}_{i}^{*}(X) \backslash \operatorname{Sing}(X)$ such that

$$
\Delta_{x}^{-}=\pi_{x}\left(\hat{E}_{x}^{s}\right) \quad \text { and } \quad \Delta_{x}^{+}=\pi_{x}^{Y}\left(\hat{E}_{x}^{u}\right), \quad \forall x \in \operatorname{Per}_{i}(X),
$$

where $T_{x} M=\hat{E}_{x}^{s} \oplus E_{x}^{X} \oplus \hat{E}_{x}^{u}$ is the corresponding hyperbolic splitting along the orbit of $x$. 
The following lemma is Theorem B in [5]. Denote by $C l(\cdot)$ the closure operation.

Lemma 2.4. If $X$ is a star flow on a closed manifold, then every compact invariant set $\Lambda \subset C l(\operatorname{Per}(X))$ with $\operatorname{Sing}(X, \Lambda)=\emptyset$ is hyperbolic.

These lemmas will be used to analyze attractors for star flows on a closed 3manifold. To start with we extend the conclusion of Lemma 2.4 to all such attractors.

Proposition 2.5. If $X$ is a star flow on a closed 3-manifold, then every attractor $A$ of $X$ with $\operatorname{Sing}(X, \Lambda)=\emptyset$ is hyperbolic.

Proof. First we show that $A \subset \operatorname{Per}_{1}^{*}(X)$ unless $A$ is a sink or a source. Indeed, if $A \not \subset \operatorname{Per}_{1}^{*}(X)$ we can select $y \in A \backslash \operatorname{Per}_{i}^{*}(X)$. As $A$ has no singularities and $y \in A$, we have that $y$ is a regular point (i.e. $X(y) \neq 0$ ). Then, it follows from Pugh's closing lemma [26] that $y \in \operatorname{Per}_{0}^{*}(X) \cup \operatorname{Per}_{2}^{*}(X)$, and so there exists a fundamental $i$-limit with $i=0,2$ intersecting $A$. As $A$ is an attractor we conclude that such a fundamental $i$-limit is contained in $A$. Therefore, by Lemma 2.1, there would exist a sink or a source contained in $A$. In such a case $A$ is a sink or a source. Then, we can assume that $A \subset \operatorname{Per}_{1}^{*}(X)$. So, Lemma 2.3 implies that there is a dominated 1-splitting $N_{A}=\Delta_{A}^{-} \oplus \Delta_{A}^{+}$for $P_{t}$ over $A$. If the subbundle $\Delta_{A}^{-}$were not contracting, there would exist a fundamental 0 -limit in $A$ by virtue of Lemma 2.2. Therefore $A$ is a source and thus hyperbolic. Hence we can assume that $\Delta_{A}^{-}$is contracting and analogously that $\Delta_{A}^{+}$is expanding. Then, $A$ is hyperbolic by Doering's criterium.

The following elementary lemma will be used to prove Proposition 2.7

Lemma 2.6. For every $\epsilon>0$ there is $\delta>0$ such that if $c:[a, b] \subset[-\epsilon, \epsilon] \rightarrow[-\epsilon, \epsilon]$ is a $C^{1}$ map satisfying

(i) $\left|c^{\prime}(t)\right| \leq \frac{1}{6}$ for all $t \in[a, b]$,

(ii) $\left|c\left(t_{0}\right)\right| \leq \delta$ for some $t_{0} \in[a, b]$,

(iii) $(a, c(a)),(b, c(b)) \in \partial\left([-\epsilon, \epsilon]^{2}\right)$,

then $a=-\epsilon, b=\epsilon$ and $|c( \pm \epsilon)|<\epsilon$.

Proof. We take $\delta=\frac{\epsilon}{3}$. Without loss of generality we can assume that $t_{0}$ in (ii) belongs to $] a, b[$. If $-\epsilon<a$, then condition (iii) implies $c(a)= \pm \epsilon$. On the other hand, condition (i) and the mean value theorem imply $\left|c\left(t_{0}\right)-c(a)\right| \leq \frac{1}{6}\left|t_{0}-a\right| \leq \frac{\epsilon}{3}$; thus (ii) yields $\epsilon \leq \frac{\epsilon}{3}+\frac{\epsilon}{3}=\frac{2 \epsilon}{3}$, which is absurd. Therefore $a=-\epsilon$, and analogously $b=\epsilon$. The same computation shows that $|c( \pm \epsilon)| \leq \frac{\epsilon}{3}$.

Hereafter we will use the standard stable and unstable manifold notation $W^{s}(\cdot)$, $W^{u}(\cdot)$ (cf. [12]).

Proposition 2.7. Let $X$ be a star flow on a closed 3-manifold and $\sigma \in \operatorname{Sing}(X)$ be such that either

(iv) $\operatorname{dim}\left(W^{s}(\sigma)\right)=2$ or

(v) $\sigma$ has three real eigenvalues $\lambda_{2}<0<\lambda_{3}<\lambda_{1}$ and $X$ cannot be $C^{1}$ approximated by vector fields with orbit-flip homoclinic orbits.

Then, for every $x \in\left(W^{s}(\sigma) \backslash\{\sigma\}\right) \cap \operatorname{Per}_{1}^{*}(X)$ there is $\delta>0$ such that $d(A, x)>\delta$ for every nontrivial hyperbolic attractor $A$ of $X$. 
Proof. Clearly $x \in \operatorname{Per}_{1}^{*}(X) \backslash \operatorname{Sing}(X)$, and so, by Lemma2.3, there is a dominated 1-splitting $N_{x}=\Delta_{x}^{-} \oplus \Delta_{x}^{+}$for $P_{t}$. It turns out that $\Delta_{x}^{-}=L_{x} \cap N_{x}$, where $L_{x}$ is either $T_{x} W^{s}(\sigma)$ or $T_{x} W^{s E}(\sigma)$ depending on whether (iv) or (v) holds. Here $W^{s E}(\sigma)$ is the extended stable manifold, i.e., the invariant manifold tangent at $\sigma$ to the eigenspace corresponding to the eigenvalues $\lambda_{2}, \lambda_{3}$ (cf. [12, [28]). Using this we can fix a cross section $\Sigma=[-\epsilon, \epsilon]^{2}$ through $x=(0,0)$ of $X$ so that:

- If (iv) holds, then $W^{s}(\sigma) \cap \Sigma$ contains the graph $\gamma=\{(u(y), y): y \in[-\epsilon, \epsilon]\}$ of a $C^{1}$ map $u:[-\epsilon, \epsilon] \rightarrow[-\epsilon, \epsilon]$ with $u(0)=0$ (cf. Figure 2(a)).

- If (v) holds, then there are $C^{1}$ maps $u_{1} \leq u_{2}:[-\epsilon, \epsilon] \rightarrow[-\epsilon, \epsilon]$ with $u_{i}(0)=u_{i}^{\prime}(0)=0($ for $i=1,2)$ such that $\operatorname{Per}_{1}^{*}(X) \cap R=\emptyset$, where $R \subset \Sigma$ is the complement of the region of $\Sigma$ in between the graphs of $\gamma_{1}$ and $\gamma_{2}$ of $u_{1}$ and $u_{2}$, respectively (i.e. the complement of the shadowed region in Figure 2(b)). These maps are obtained by pushing backward a cross-section through a suitable weak unstable manifold of $\sigma$.

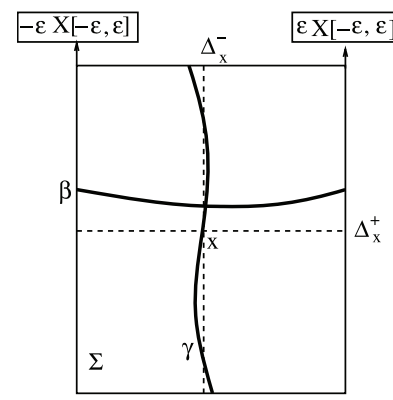

(a)

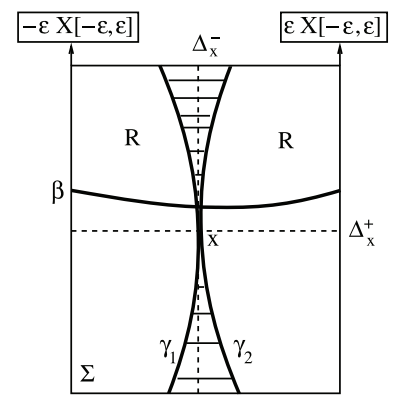

(b)

FIGURE 2

Shrinking $\epsilon$ if necessary we can assume that any $C^{1}$ map $c:[a, b] \subset[-\epsilon, \epsilon] \rightarrow$ $[-\epsilon, \epsilon]$ whose graph $\gamma=\left\{(t, c(t): t \in[a, b]\}\right.$ is tangent to $\Delta_{P e r_{1}^{*}(X) \cap \Sigma}^{+}$satisfies hypothesis (i) in Lemma 2.6 i.e., $\left|c^{\prime}(t)\right| \leq \frac{1}{6}$ for all $t \in[a, b]$.

Now, take $\delta>0$ as in Lemma 2.6 for such an $\epsilon$ and a nontrivial hyperbolic attractor $A$ with $d(A, x) \leq \delta$. Then, using a tubular flow box around $x$ we have that there is $y \in A \cap \Sigma$ with $d(x, y) \leq \delta$.

Let $\beta$ be the connected component of $W^{u}(y) \cap \Sigma$ containing $y$. Since $A$ is a nontrivial hyperbolic attractor, standard facts about hyperbolic sets (e.g. the local product structure [9]) imply that the end points of $\beta$ belong to $\partial \Sigma$. Moreover, $A \subset \operatorname{Per}_{1}^{*}(X) \backslash \operatorname{Sing}(X)$ (by the shadowing lemma for flows [9]) and, since $\Delta_{x}^{+}=$ $\pi_{x}\left(\hat{E}_{x}^{u}\right)$ for $x \in \operatorname{Per}_{1}(X)$ (by Lemma 2.3) and the periodic orbits in $A$ are dense in $A$, we obtain that $\beta$ is tangent to $\Delta_{P e r_{1}^{*}(X) \cap \Sigma}^{+}$. Then, $\beta$ is the graph of a $C^{1}$ map $c:[a, b] \rightarrow[-\epsilon, \epsilon]$ with $c\left(t_{0}\right)=y$ for some $t_{0} \in(a, b)$, and so $c$ satisfies hypotheses (i) and (ii) of Lemma 2.6. Additionally, since the end points of $\beta$ belong to $\partial \Sigma$, we also have $c(a), c(b) \in \partial(\Sigma)$, and so $c$ also satisfies hypothesis (iii) of Lemma 2.6. Then, Lemma 2.6 implies $a=-\epsilon$ and $b=\epsilon$ and $|c( \pm \epsilon)|<\epsilon$. Consequently, $\beta$ joins $-\epsilon \times[-1,1]$ to $\epsilon \times[-1,1]$, as indicated in Figure 2 , 
If (iv) holds, then $\beta$ (which is contained in $A$ ) intersects $\gamma$ (which is contained in $W^{s}(\sigma)$ ), whence $\sigma \in A$, which is absurd since $A$ is a nontrivial hyperbolic attractor. Therefore, (v) holds, and so $\beta \cap R \neq \emptyset$, yielding $\operatorname{Per}_{1}^{*}(X) \cap R \neq \emptyset$, again an absurdity. These contradictions prove the result.

Now we prove the following key result.

Proposition 2.8. Let $X$ be a star flow with singularities on a closed 3-manifold which cannot be $C^{1}$ approximated by vector fields with orbit-flip homoclinic orbits. Then, there is a neighborhood $U$ of $\operatorname{Sing}(X)$ such that if $A$ is an attractor of $X$, then $A \cap U \neq \emptyset$ if and only if $\operatorname{Sing}(X, A) \neq \emptyset$.

Proof. Otherwise there is a sequence of attractors $A_{n}$ with $\operatorname{Sing}\left(X, A_{n}\right)=\emptyset$ and $\sigma \in \Lambda \cap \operatorname{Sing}(X)$, where

$$
\Lambda=C l\left(\bigcup_{n} A_{n}\right) .
$$

Since star flows have finitely many sinks ([15], 25]) we can assume that each $A_{n}$ is nontrivial and they are all hyperbolic by Proposition 2.5. It follows that every $A_{n}$ is a nontrivial hyperbolic attractor, and so $\Lambda \subset \operatorname{Per}_{1}^{*}(X)$.

We clearly have that $\sigma$ is neither a sink nor a source (otherwise it could not be accumulated by periodic orbits, which is the case for $\sigma$ ). So, we can order its eigenvalues $\lambda_{1}, \lambda_{2}, \lambda_{3}$ in a way that either $\lambda_{2}$ or $\lambda_{1}$ is real and, in each case,

$$
\operatorname{Re}\left(\lambda_{2}\right) \leq \operatorname{Re}\left(\lambda_{3}\right)<0<\lambda_{1} \quad \text { or } \quad \lambda_{2}<0<\operatorname{Re}\left(\lambda_{3}\right) \leq \operatorname{Re}\left(\lambda_{1}\right),
$$

with $R e(\cdot)$ denoting the real part.

In the first case $\sigma$ clearly satisfies hypothesis (iv) of Proposition 2.7. In the second we must have that both $\lambda_{3}$ and $\lambda_{1}$ are real (otherwise the dominated 1splitting claimed to exist in Lemma 2.3 would not exist), and since $X$ cannot be approximated by vector fields with orbit-flip homoclinic loops, we still have $\lambda_{3}<\lambda_{1}$. In other words, in such a case $\sigma$ satisfies hypothesis (v) of Proposition 2.7. On the other hand, in both cases it is certainly possible to find $x \in\left(W^{s}(\sigma) \backslash\{\sigma\}\right) \cap \Lambda$. In particular, $x \in \operatorname{Per}_{1}^{*}(X) \backslash \operatorname{Sing}(X)$, and so, by Proposition 2.7, there is $\delta>0$ such that $d\left(A_{n}, x\right) \geq \delta$ for all $n$. But this is clearly impossible due to the definition of $\Lambda$, so the result is true.

Proof of the Main Theorem. Let $X$ be a star flow on a closed 3-manifold which cannot be $C^{1}$ approximated by vector fields exhibiting orbit-flip homoclinic orbits. We can assume without any loss of generality that $X$ has singularities (if not, we apply [5]). Suppose by contradiction that it has infinitely many distinct attractors $A_{n}, n \in \mathbb{N}$. Since $X$ has finitely many singularities and sinks and since the attractors are pairwise disjoint, we can assume that each $A_{n}$ is not a sink and satisfies $\operatorname{Sing}\left(X, A_{n}\right)=\emptyset$. In particular, each $A_{n}$ is a nontrivial hyperbolic attractor by Proposition [2.5. Moreover, by Proposition 2.8, there is a neighborhood $U$ of $\operatorname{Sing}(X)$ such that $A_{n} \cap U=\emptyset$ for all $n$. It follows that the closure $C l\left(\bigcup_{n} A_{n}\right)$ has no singularities. Since each $A_{n}$ is a nontrivial hyperbolic attractor, we have $A_{n} \subset C l(\operatorname{Per}(X))$, and so $C l\left(\bigcup_{n} A_{n}\right)$ is also a compact invariant set in $C l(\operatorname{Per}(X))$. Applying Lemma 2.4 we conclude that $C l\left(\bigcup_{n} A_{n}\right)$ is a hyperbolic set. However, as is well known, hyperbolic sets contain only a finite number of attractors, which is certainly not the case for $C l\left(\bigcup_{n} A_{n}\right)$. Thus we obtain a contradiction, which proves the result. 


\section{REFERENCES}

[1] Afraimovich, V. S., Bykov, V. V., Shilnikov, L. P., On attracting structurally unstable limit sets of Lorenz attractor type (Russian), Trudy Moskov. Mat. Obshch. 44 (1982), 150-212. MR656286(84a:58058)

[2] Aoki, N., The set of Axiom A diffeomorphisms with no cycles, Bol. Soc. Brasil. Mat. (N.S.) 23 (1992), no. 1-2, 21-65. MR1203172 (94d:58080)

[3] Champneys, A. R., Homoclinic orbits in reversible systems and their applications in mechanics, fluids and optics, Time-reversal symmetry in dynamical systems (Coventry, 1996). Phys. D 112 (1998), no. 1-2, 158-186. MR1605836 (99b:58169)

[4] Doering, C. I., Persistently transitive vector fields on three-dimensional manifolds, Dynamical systems and bifurcation theory (Rio de Janeiro, 1985), 59-89, Pitman Res. Notes Math. Ser., 160, Longman Sci. Tech., Harlow, 1987. MR907891 (89c:58111)

[5] Gan, S., Wen, L., Nonsingular star flows satisfy Axiom A and the no-cycle condition, Invent. Math. 164 (2006), no. 2, 279-315. MR2218778 (2007j:37045)

[6] Gan, S., Li, M., Wen, L., Robustly transitive singular sets via approach of an extended linear Poincaré flow, Discrete Contin. Dyn. Syst. 13 (2005), no. 2, 239-269. MR2152388 (2006b:37056)

[7] Guckenheimer, J., A strange, strange attractor, The Hopf bifurcation and its applications, Applied Mathematical Series, 19, Springer-Verlag, 1976. MR0494309 (58:13209)

[8] Guckenheimer, J., Williams, R., Structural stability of Lorenz attractors, Publ. Math. IHES 50 (1979), 59-72. MR556582 (82b:58055a)

[9] Hasselblatt, B., Katok, A., Introduction to the modern theory of dynamical systems. With a supplementary chapter by Katok and Leonardo Mendoza, Encyclopedia of Mathematics and its Applications, 54, Cambridge University Press, Cambridge, 1995. MR.1326374 (96c:58055)

[10] Hayashi, S., Connecting invariant manifolds and the solution of the $C^{1}$ stability and $\Omega$ stability conjectures for flows, Ann. of Math. (2) 145 (1997), no. 1, 81-137. MR1432037 (98b:58096)

[11] Hayashi, S., Diffeomorphisms in $\mathcal{F}^{1}(M)$ satisfy Axiom A, Ergodic Theory Dynam. Systems 12 (1992), no. 2, 233-253. MR1176621 (94d:58081)

[12] Hirsch, M., Pugh, C., Shub, M., Invariant manifolds, Lecture Notes in Mathematics, Vol. 583. Springer-Verlag, Berlin-New York, 1977. MR0501173 (58:18595)

[13] Homburg, A. J., Krauskopf, B., Resonant homoclinic flip bifurcations, J. Dynam. Differential Equations 12 (2000), no. 4, 807-850. MR1826963 (2002h:37089)

[14] Kokubu, H., Komuro, M., Oka, H., Multiple homoclinic bifurcations from orbit-flip. I. Successive homoclinic doublings, Internat. J. Bifur. Chaos Appl. Sci. Engrg. 6 (1996), no. 5, 833-850. MR 1404121 (97e:58168)

[15] Liao, S., Qualitative theory of differentiable dynamical systems. Translated from the Chinese. With a preface by Min-de Cheng. Science Press, Beijing; distributed by American Mathematical Society, Providence, RI, 1996. MR1449640 (98g:58041)

[16] Lopes, A. O., Structural stability and hyperbolic attractors, Trans. Amer. Math. Soc. 252 (1979), 205-219. MR534118 (80j:58046)

[17] Mañé, R., A proof of the $C^{1}$ stability conjecture, Inst. Hautes Études Sci. Publ. Math., No. 66 (1988), 161-210. MR932138(89e:58090)

[18] Mañé, R., Contributions to the stability conjecture, Topology 17 (1978), no. 4, 383-396. MR516217 (84b:58061)

[19] Morales, C. A., The explosion of singular-hyperbolic attractors, Ergodic Theory Dynam. Systems 24 (2004), no. 2, 577-591. MR2054194 (2005d:37071)

[20] Morales, C. A., Pacifico, M. J., A dichotomy for three-dimensional vector fields, Ergodic Theory Dynam. Systems 23 (2003), no. 5, 1575-1600. MR2018613 (2005a:37030)

[21] Morales, C. A., Pacifico, M. J., Lyapunov stability of $\omega$-limit sets, Discrete Contin. Dyn. Syst. 8 (2002), no. 3, 671-674. MR.1897874 (2003b:37024)

[22] Morales, C. A., Pacifico, M. J., Inclination-flip homoclinic orbits arising from orbit-flip, Nonlinearity 14 (2001), no. 2, 379-393. MR1819803 (2001m:37103)

[23] Naudot, V., A strange attractor in the unfolding of an orbit-flip homoclinic orbit, Dyn. Syst. 17 (2002), no. 1, 45-63. MR.1888697(2002m:37032)

[24] Palis, J., Open questions leading to a global perspective in dynamics, Nonlinearity 21 (2008), no. 4, T37-T43. MR2399817 (2009i:37003) 
[25] Pliss, V. A., A hypothesis due to Smale, Differencial'nye Uravnenija 8 (1972), 268-282. MR0299909 (45:8957)

[26] Pugh, C., An improved closing lemma and a general density theorem, Amer. J. Math. 89 (1967), 1010-1021. MR0226670 (37:2257)

[27] Ruelle, D., Takens, F., On the nature of turbulence, Comm. Math. Phys. 20 (1971), 167-192. MR0284067 (44:1297)

[28] Shilnikov, L. P., Shilnikov, A. L., Turaev, D., Chua, L., Methods of qualitative theory in nonlinear dynamics. Part II, World Scientific Series on Nonlinear Science. Series A: Monographs and Treatises, 5. World Scientific Publishing Co., Inc., River Edge, NJ, 2001. MR 1884710 (2003d:37002)

[29] Turaev, D., Multi-pulse homoclinic loops in systems with a smooth first integral, Ergodic theory, analysis, and efficient simulation of dynamical systems, 691-716, Springer, Berlin, 2001. MR1850326 (2002f:37094)

[30] Wen, L., On the preperiodic set, Discrete Contin. Dynam. Systems 6 (2000), no. 1, 237-241. MR.1739926 (2001g:37035)

[31] Wen, L., On the $C^{1}$ stability conjecture for flows, J. Differential Equations 129 (1996), no. 2, 334-357. MR 1404387 (97j:58082)

Instituto de Matemática, Universidade Federal do Rio de Janeiro, P. O. Box 68530, 21945-970 Rio de JANEIRo, BraziL

E-mail address: morales@impa.br 